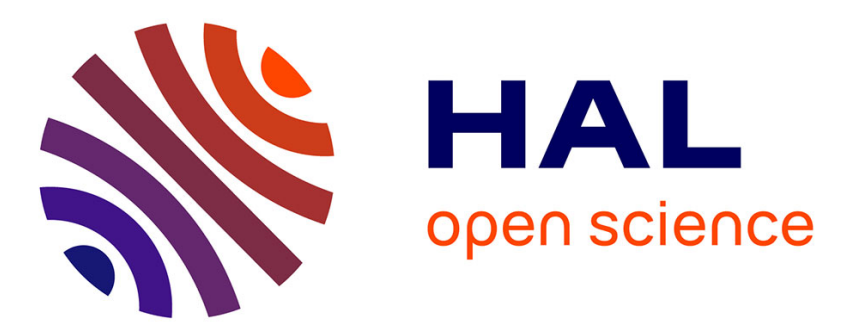

\title{
Geometric study of a 2D tiling related to the octagonal quasiperiodic tiling
}

Clément Sire, Rémy Mosseri, Jean-François Sadoc

\section{To cite this version:}

Clément Sire, Rémy Mosseri, Jean-François Sadoc. Geometric study of a 2D tiling related to the octagonal quasiperiodic tiling. Journal de Physique IV Proceedings, 1989, 50 (24), pp.3463-3476. 10.1051/jphys:0198900500240346300 . hal-02382037

\section{HAL Id: hal-02382037 \\ https://hal.science/hal-02382037}

Submitted on 27 Nov 2019

HAL is a multi-disciplinary open access archive for the deposit and dissemination of scientific research documents, whether they are published or not. The documents may come from teaching and research institutions in France or abroad, or from public or private research centers.
L'archive ouverte pluridisciplinaire HAL, est destinée au dépôt et à la diffusion de documents scientifiques de niveau recherche, publiés ou non, émanant des établissements d'enseignement et de recherche français ou étrangers, des laboratoires publics ou privés. 


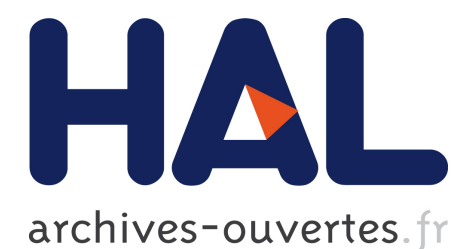

\section{- To cite this version:}

Clément Sire, Rémy Mosseri, Jean-François Sadoc. Geometric study of a 2D tiling related to the octagonal quasiperiodic tiling. Journal de Physique, 1989, 50 (24), pp.3463-3476. $<10.1051 /$ jphys:0198900500240346300 > . <jpa-00211156>

\section{HAL Id: jpa-00211156 \\ https://hal.archives-ouvertes.fr/jpa-00211156}

Submitted on 1 Jan 1989

HAL is a multi-disciplinary open access archive for the deposit and dissemination of scientific research documents, whether they are published or not. The documents may come from teaching and research institutions in France or abroad, or from public or private research centers.
L'archive ouverte pluridisciplinaire HAL, est destinée au dépôt et à la diffusion de documents scientifiques de niveau recherche, publiés ou non, émanant des établissements d'enseignement et de recherche français ou étrangers, des laboratoires publics ou privés. 
Classification

Physics Abstracts

$61.50 \mathrm{E}$

\title{
Geometric study of a 2D tiling related to the octagonal quasiperiodic tiling
}

\author{
Clément Sire $\left({ }^{1}\right)$, Rémy Mosseri $\left({ }^{1}\right)$ and Jean-François Sadoc $\left({ }^{2}\right)$ \\ (1) Laboratoire de Physique des Solides de Bellevue, C.N.R.S., 1 place Aristide Briand, 92195 \\ Meudon, France \\ (2) Laboratoire de Physique des Solides, Université Paris-Sud, 91405 Orsay, France
}

(Reçu le 31 mai 1989, révisé le 19 juillet 1989, accepté le 6 septembre 1989)

\begin{abstract}
Résumé. - Au moyen de trois tuiles, nous construisons un pavage quasipériodique du plan, que nous relions au quasicristal octogonal. Ainsi, nous montrons que les coordonnées des nœuds peuvent être obtenues de deux manières différentes. Le facteur de structure est calculé exactement. Ce pavage qui possède "presque » une symétrie d'ordre huit, soulève la difficulté de la détermination pratique de la symétrie d'un quasicristal. Finalement, nous montrons comment construire une large classe de pavage du type de l'octogonal, à partir de ce nouveau pavage.

Abstract. - A quasicrystal built with three types of tiles is related to the well-known octagonal tiling. The relationships between both tilings are investigated. More precisely, we show that the coordinates of the vertices can be obtained in two different but equivalent ways. The structure factor is calculated exactly. We emphazise the difficulty one can have to define the order of the symmetry of a quasicrystal, from a practical point of view, exhibiting a quasiperiodic tiling whose spectrum has a « quasi » eigth-fold symmetry. Finally, we show how to recover easily a class of octagonal-like quasicrystals.
\end{abstract}

\section{Introduction.}

Since the discovery of alloys with quasiperiodic structure by Shechtman et al. [1], many cares have been devoted in the experimental domain, to the determination of atomic positions. This is a prerequisite to a better understanding of physical properties. At the theory level, a parallel effort has been done with many new results concerning the geometry of the quasiperiodic structures and their diffraction patterns. Concerning the physical properties, however, the field is still in its infancy. This is mainly due to the lack of appropriate tools, the analogues for instance, of Bloch sums, or Brillouin zones of crystal physics. Only in one dimension do we have a rather precise knowledge of the quasicrystal excitation spectrum.

This is why we found interesting to look to extensions to higher dimensions of these 1D quasicrystals. In the case of the $1 \mathrm{D}$ structure related to the irrational number $1+\sqrt{2}$, we have discovered a more interesting tiling, the "labyrinth ", which is a subset of order two of the euclidian product. As will be shown below, the labyrinth is very closely related to the standard octagonal $[5,6]$ quasiperiodic tiling. However, it is much simpler to many respects. For 
example, its coordinates can be given explicitely, which is a non trivial property for quasiperiodic tilings of dimension greater than 1 . This tiling has a very simple average lattice, and their mutual correspondence is derived in a straightforward way from the expression of the site coordinates.

\section{The recursive method.}

We shall consider a sequence of letters $\mathrm{A}$ and $\mathrm{B}$ describing the alternation of segments of two different lengths. Let us call $S_{\ell}$ the sequence obtained according to the following rule :

$$
S_{\ell+2}=S_{\ell+1} * S_{\ell} * S_{\ell+1} \quad S_{0}=B, \quad S_{1}=A, \quad S_{\infty}=\lim _{\ell \infty} S_{\ell} .
$$

where $*$ is a symbol for string concatenation. This sequence is of the same kind as the wellknown Fibonacci sequence studied in a very exhaustive way by many authors. This sequence, which we shall call the "Octonacci » sequence, is related to $\Phi=1+\sqrt{2}$ in the following way: if $F_{\ell}$ is defined as the length of $S_{\ell}$ then $F_{\ell}$ obeys the same recursion formula as $S_{\ell}$ with $F_{0}=F_{1}=1$. Then, it is easy to see that $F_{\ell+1} / F_{\ell} \rightarrow \Phi$ when $\ell \rightarrow+\infty$. Moreover, $S_{\ell+1}$ can be obtained from $S_{\ell}$ by applying the following rule due to the self-similarity of $S_{\infty}$ :

$$
\mathrm{A} \rightarrow \mathrm{ABA}, \quad \mathrm{B} \rightarrow \mathrm{A}
$$

It is well-known that $S_{\infty}$ is nor periodic neither random but quasiperiodic. Then, a sequence of arbitrarily length in $S_{\infty}$ can be found an infinite number of times.

Now, after having associated a length $\ell_{\mathrm{A}}$ to an $\mathrm{A}$ and a length $\ell_{\mathrm{B}}$ to each $\mathrm{B}$, let us define the «labyrinth» tiling by selecting among the set of points of the Euclidian product $S_{\ell} \times S_{\ell}$, those sites which can be linked to their neighboring sites by a path of non vertical nor horizontal bonds, starting the process at the origin (Fig. 1). More precisely, we see that this tiling consists of half the whole product set. Finally, if we join the nearest neighbors by

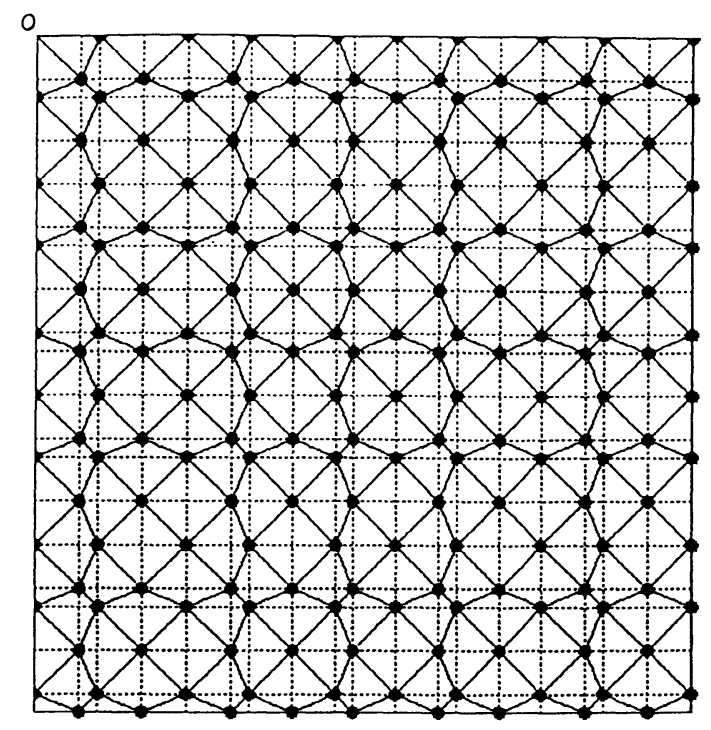

Fig. 1. - The labyrinth is obtained starting from the euclidian product $S_{\ell} \times S_{\ell}$ (dotted lines) by linking points with diagonal bonds. 
« diagonal » bonds, we obtain a tiling of one quarter of the plane. In order to obtain a tiling of the whole plane, we tile the three other quarters in the same way as the first one. The infinite tiling we obtain is of course quasiperiodic and consists in the assembly of three elementary tiles whose exact shapes depend on the ratio $r=\ell_{\mathrm{A}} / \ell_{\mathrm{B}}$. We always obtain a square, a trapezoid and a kite whose sides are given on figure 2 . If we colour each elementary tile in a different way, the reason for calling this tiling, the «labyrinth », becomes manifest. We show on figure 3 the labyrinth for $r<1$ and $r>1$. We can even consider the limit case $r=1$, and obtain a quasiperiodically coloured square lattice ressemling a labyrinth (Fig. 3). If we follow one of those paths, we can see that it can be arbitrarily long. Indeed, since $S_{\infty}$ is self-similar we expect the «labyrinth " to be self-similar too. We found inflationdeflation rules for this quasiperiodic tiling which we have summarized in figure 2 The shapes of the inflated tiles are the same as those of the original tiling if and only if $r=1+\sqrt{2}=\Phi$. It is possible to do the same procedure starting with the Fibonacci sequence or any other sequence not related to $\Phi$. Note that the two 1D quasicrystals, are not necessarily the same, neither at right angle. In this paper, we focus however, on the structure based on the Octonacci sequence, and first describe another construction of the labyrinth.
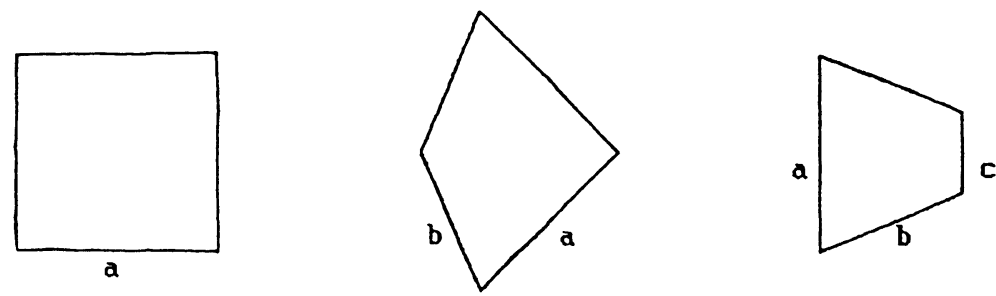

a)
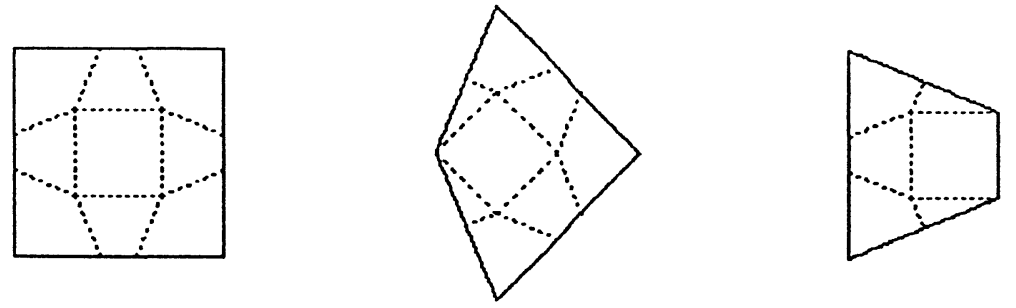

b)
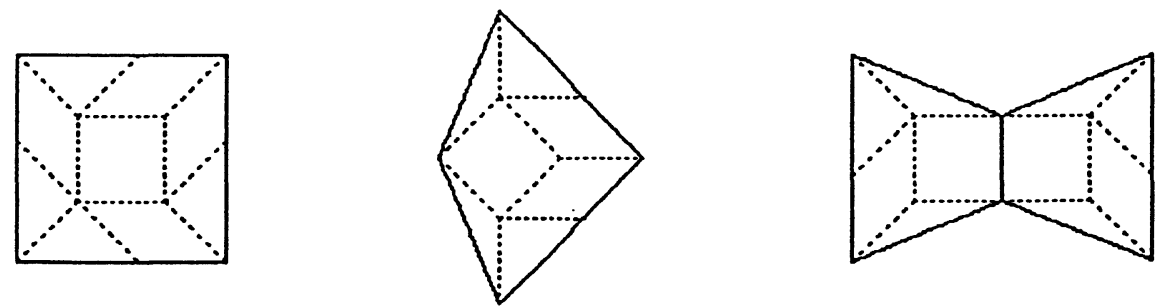

c)

Fig. 2. - (a) The labyrinth is made of three elementary tiles : a square, a kite, and a trapezoid. The lengths $a, b, c$, are respectively equal to $\sqrt{2 \ell_{A}}, \sqrt{\left(\ell_{A}^{2}+\ell_{B}^{2}\right)}$ and $\sqrt{2 \ell_{B}}$. Here we took $r=\ell_{\mathrm{A}} / \ell_{\mathrm{B}}=1+\sqrt{2}$. (b) Deflation rules for the labyrinth. (c) The decorations of the three tiles leading to the octogonal quasiperiodic tiling. 


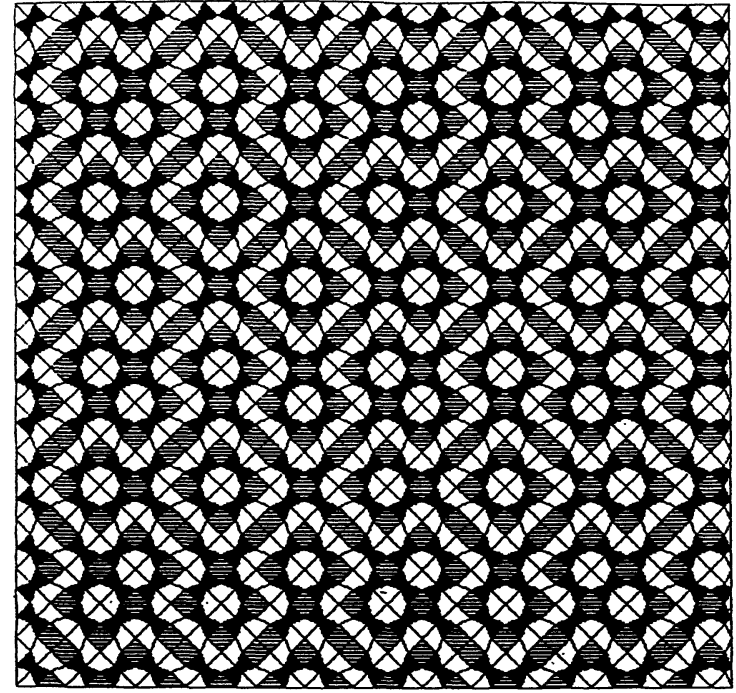

a)

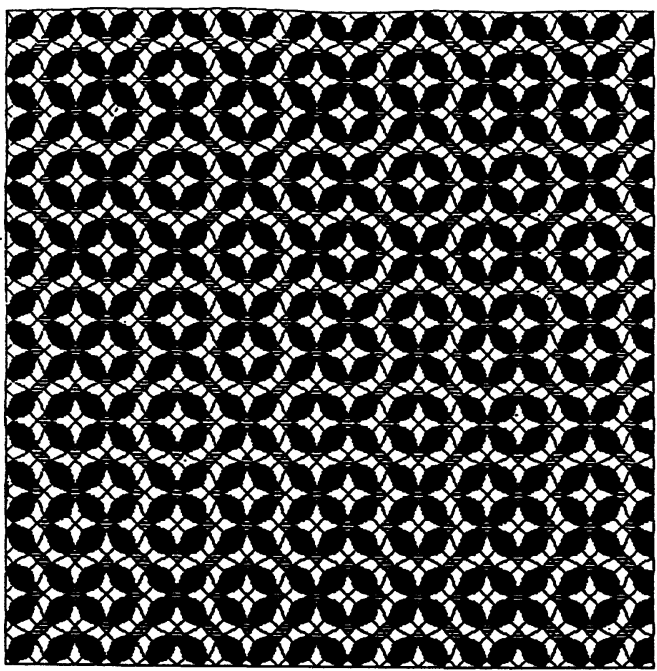

b)

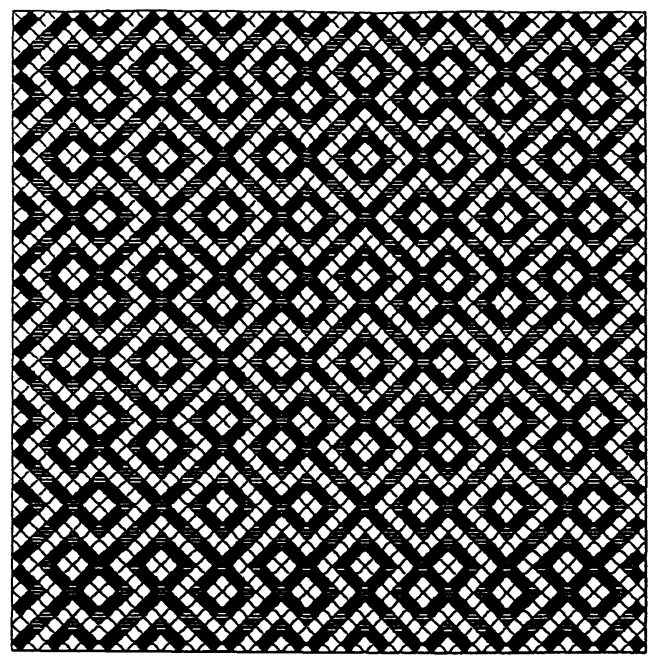

c)

Fig. 3. - The labyrinth for (a) $r=\Phi$, (b) $r=1 / \Phi$, and (c) $r=1$.

3. Another derivation of the labyrinth.

The labyrinth structure appears very simply out of a new construction of quasiperiodic tilings [2], derived from the cut and project method [4]. This method is rather general but we shall focus on the octagonal tiling case. Vertices of the hypercubic lattice $Z^{4}$ are selected and mapped onto a $2 \mathrm{D}$ physical space $\mathrm{E}$, whenever their projection onto the $2 \mathrm{D}$ space $\mathrm{E}^{\prime}$ (orthogonal to $\mathrm{E}$ ) falls inside a window $\mathrm{W}$. The window is the convex hull of the projection onto $\mathrm{E}^{\prime}$ of the standard hypercube of $Z^{4}$. Now, let us view $Z^{4}$ as a stratified structure : a 2D square lattice (the base) of $2 \mathrm{D}$ square lattices (the fibres), associated to each point of the base. 
The acceptance region can be drawn on each fibre as the projection, parallel to $\mathrm{E}$, of the unit hypercube. The octagonal tiling vertices can then be selected. The procedure can be done directly on $\mathrm{E}$ by mapping these acceptance regions, one for each fibre. The projection onto $\mathrm{E}$ of the base gives a square lattice $B$. The acceptance regions are regular octogons $\mathrm{O}_{\ell_{m}}$, centered on a sublattice of order 4 of $\mathrm{B}$. To each point $\mathrm{B}_{\ell_{m}}$, the corresponding $\mathrm{O}_{\ell_{m}}$ is centered on the line from the origine to $\mathrm{B}_{\ell_{m}}$, at twice the distance. Each fibre projects onto $\mathrm{E}$ as another square lattice $F_{\ell, m}$, identical to $\mathrm{B}$, but rotated by $\pi / 4$. The vertices are selected as follows. To each point $\mathrm{B}_{\ell_{m}}$, one attaches a square lattice $F_{\ell, m}$, from which we select those vertices which fall inside the octogon $\mathrm{O}_{\ell_{m}}$. An octagonal tiling is thus recovered. But instead, if in each octogon one only keeps the vertex of $F$ closest to the $\mathrm{O}_{\ell_{m}}$ center, which we call $\mathrm{Q}_{\ell_{m}}$, then, the labyrinth is obtained. The coordinates can be easily obtained. Let us derive them in the B-basis with unit edge. We have

$$
\mathrm{B}=\{(\ell, m) / \ell, m \in \mathbf{Z}\} \quad \text { and } \quad \mathrm{Q}_{\ell, m}=(2 \ell, 2 m) .
$$

Let $A$ be the rotation matrix by $\pi / 4$

$$
A=1 / \sqrt{2}\left(\begin{array}{rr}
1 & -1 \\
1 & 1
\end{array}\right)
$$

So, the lattice $F$ is obtained by applying $A$ to each vertex of $\mathrm{B}$. In the $F$ basis, the point $\mathrm{B}_{\ell_{m}}$ has coordinates given by $A^{-1}\left(\mathbf{B}_{\ell_{m}}\right)$. In order to attach a $F$ lattice to $\mathrm{B}_{\ell_{m}}$, one should shift it by a vector $S_{\ell_{m}}$

$$
S_{\ell_{m}}=\operatorname{frac}\left(A^{-1}\left(\mathrm{~B}_{\ell_{m}}\right)\right)
$$

where $\operatorname{frac}(x)$ is the fractional part of the coordinates of $x$. In the shifted $F$ basis, the point $\mathrm{Q}_{\ell_{m}}$ has coordinates equal to $A^{-1}\left(\mathrm{Q}_{\ell_{m}}\right)-S_{\ell_{m}}$. The point of the shifted $F$ closest to $\mathrm{Q}_{\ell_{m}}$ is

$$
F\left(A^{-1}\left(\mathrm{Q}_{\ell_{m}}\right)-S_{\ell_{m}}\right)
$$

where $F(x)$ is the closest integer to $x$. In the B-basis, the coordinates are

$$
\left(X_{\ell_{m}}, Y_{\ell_{m}}\right)_{\mathrm{B}}=A\left(F\left(A^{-1}\left(\mathrm{Q}_{\ell_{m}}\right)+S_{\ell_{m}}\right)+S_{\ell_{m}}\right) \text {. }
$$

This can be written

$$
\begin{array}{ll}
X_{\ell_{m}}=\ell+a(F(a(\ell+m))+F(a(\ell+m))) & (\ell, m) \in Z^{2} \\
Y_{\ell m}=m+a(F(a(\ell+m))-F(a(\ell-m))) & a=1 \sqrt{2} .
\end{array}
$$

These above relations form what is called explicit coordinates for the tiling vertices. It is a one to one relation between a couple of integers $(\ell, m)$ which takes all possible values, and the corresponding pair of real coordinates. This is not the case for tilings generated by the standard cut and project method. In that case, the vertex coordinates can be written as a linear combination (with integer coefficients) of a set of $n$ "star " vectors. But among all possible $n$-uplets, only a few are kept after a selection procedure in the orthogonal space. It is precisely this algorithmic step (the most time consuming) which is bypassed when explicit coordinates are given.

Now, consider a non periodic array of points. It is said to possess an average (periodic) lattice if there exists a one-to-one relation between the former points and the lattice points, and if their distance remains bounded. With this definition, the labyrinth has an average 
square lattice, the acceptance region lattice, which shows up simply by replacing $F(x)$ by an identity operator. This is not surprising since the octagonal tiling itself was found to have an average lattice [3]. This question of non periodic tilings having or not an average lattice is a difficult one and is in fact two fold. First is the existence problem. A surprising result comes from the study of $1 \mathrm{D}$ tilings generated by the circle map [7]. As a consequence of a theorem by Kesten, it is found that for a large class of such tilings, the fluctuation of the site coordinates from their average position (the lattice defined by the density) is unbounded. Other results, in the context of the cut and project method, have been obtained [8] which show that it is a subtle question. The second point is, whenever an average lattice exists, to find the explicit one-to-one relation. In the case of the octogonal tiling, this is a difficult task. In the present labyrinth case, both the existence and the explicit relation can be very simply read from the above expressions. From the more physical point of view, it may be argued that viewing a quasicrystal as a suitably distorted crystal can open new field of investigations. As an indication among others, the Hume-Rothery type of arguments to explain their relative stability [9] can then be studied. For example in 1D, it has been shown that the Fibonacci chain might be stabilized with respect to the periodic chain for some electron densities [10].

\section{Proof of the equivalence of the two methods.}

In the following, we show that the preceding definition of the labyrinth is equivalent to the one given in section 2 . Let us define

$$
p=\ell+m, q=\ell-m \text { or } \ell=(p+q) / 2, \quad m=(p-q) / 2 .
$$

We notice that $p$ and $q$ have the same parity. Therefore, it is equivalent to define the labyrinth by the points whose coordinates are

$$
X_{p q}=U_{p}+U_{q} \quad Y_{p q}=U_{p}-U_{q} \quad p \equiv q[2]
$$

and

$$
U_{p}=p / 2+a F(a p) .
$$

With this new definition and since $U_{p}-U_{p-\ell}$ takes only two values $1 / 2$ and $1 / 2+a$, in order to show that both definitions are equivalent, we only need to prove that the sequence of 0 and 1 defined by

$$
R_{\ell}(p)=\left(U_{p}-U_{p-1}-1 / 2\right) / a \quad p=1,2, \ldots, F_{\ell}
$$

where $R_{\ell}(p)$ is the $p^{\text {th }}$ term of the sequence of length $F_{\ell}$, is equal to $S_{\ell}$ with the correspondence

$$
A \leftrightarrow 0 \quad \text { and } \quad B \leftrightarrow 1
$$

First of all, we define a sequence of rational approximants for $a, p_{\ell} / q_{\ell} \ell \in N$. $p_{\ell}$ and $q_{\ell}$ verify the following recursion formulas

$$
\begin{array}{lll}
p_{\ell+2}=2 p_{\ell+1}+p_{\ell} & p_{0}=0 & p_{1}=1 \\
q_{\ell+2}=2 q_{\ell+1}+q_{\ell} & q_{0}=1 & q_{1}=1
\end{array}
$$

since all the terms in the continued fraction representing $a$ are equal to 2 . The first step of the proof consists in showing that for $\delta$ small enough, $F\left(q p_{\ell} / q_{\ell}+\delta\right)=F\left(q p_{\ell} / q_{\ell}\right)$. For any integers $p$ and $q$, and for $\theta= \pm 1$, we have $2 q p_{\ell}-(2 p+\theta) q_{\ell} \neq 0$, since for any $\ell$, it is easy to show that $q_{\ell}$ is odd. Therefore, we obtain the inequality

$$
\left|q p_{\ell} / q_{\ell}-p+\theta / 2\right| \geqslant 1 / 2 q_{\ell} \quad p, q \in \mathrm{Z} \quad \theta= \pm 1 .
$$


Thus, if $q=1,2, \ldots, q_{\ell}=F_{\ell}$, and if $p=F\left(q p_{\ell} / q_{\ell}\right)$, we can write

$$
q p_{\ell} / q_{\ell}=p+d \quad|d|<\left(1-1 / q_{\ell}\right) / 2 .
$$

This last inequality shows that

$$
F\left(q p_{\ell} / q_{\ell}+\delta\right)=F\left(q p_{\ell} / q_{\ell}\right) \quad \text { for } \quad|\delta|<1 / 2 q_{\ell}
$$

since

$$
|d+\delta|<1 / 2 \text {. }
$$

Now, using this last result, it becomes easy to see that $F(a q)=F\left(q p_{\ell+2} / q_{\ell}+2\right)$ for any $q=1, \ldots, q_{\ell+2}$ by recalling that, according to the definition of $p_{\ell+2}$ and $q_{\ell+2}$, we have $\left|a-p_{\ell+2} / q_{\ell+2}\right|<1 / q_{\ell}+2 q_{\ell+3}$. We can therefore rewrite

$$
R_{\ell}(q)=F\left(q p_{\ell+2} / q_{\ell+2}\right)-F\left((q-1) p_{\ell+2} / q_{\ell+2}\right) .
$$

We deduce that $R_{\ell}(q)=R_{\ell}\left(q_{\ell+2}-q+1\right)$ and thus, that $R_{\ell}$ is symmetric. Since $R_{\ell+2}$ is beginning in the same way as $R_{\ell+1}$, we have shown that

$$
R_{\ell+2}=R_{\ell+1} * W_{\ell} * R_{\ell+1}
$$

where $W_{\ell}$ is a sequence of length $q_{\ell}=F_{\ell}$. We end this proof by showing that $W_{\ell}=R_{\ell}$. For $q=1,2, \ldots, q_{\ell}$, we have to show that

$$
R_{\ell+2}\left(q_{\ell+1}+q\right)=R_{\ell}(q) .
$$

This is a consequence of the first lemma we proved in the beginning of this section and the following property verified by $p_{\ell} / q_{\ell}$ :

$$
\begin{aligned}
\left(q+q_{\ell+1}\right) p_{\ell+2} / q_{\ell+2} & =q p_{\ell} / q_{\ell}+(-1)^{\ell}\left(2 q / q_{\ell}-1\right) / q_{\ell+2}+p_{\ell+1} \\
& =q p_{\ell} / q_{\ell}+p_{\ell+1}+\delta_{1} \text { with }\left|\delta_{1}\right| \leqslant 1 q_{\ell+2}<q_{\ell+2}<1 / 2 q_{\ell}
\end{aligned}
$$

where we used the fact that

$$
p_{\ell+2} q_{\ell}-p_{\ell} q_{\ell+2}=2(-1)^{\ell} \quad \text { and } \quad p_{\ell+2} q_{\ell+1}-p_{\ell+1} q_{\ell+2}=(-1)^{\ell} .
$$

Moreover, since $R_{0}=S_{0}, R_{1}=S_{1}$ and $R_{\ell}$ and $S_{\ell}$ obey the same recursion formula we conclude that they are equal, and thus, that both definitions for the coordinates of the vertices of the labyrinth are completely equivalent.

Let us show now, two other ways to build the labyrinth. The first one consists in starting from an octagonal quasicrystal. But instead of applying the process described in section 3 , we take as the vertices of the labyrinth, the vertices of the octagonal tiling, plus some new points choosen in the following way. Each horizontal square of the octagonal tiling is surrounded at least by two rhombi, and forms an hexagon with them. We add a vertex and two bonds in each such hexagon, symmetric to the already existing vertex and bonds, with respect to the center of the hexagon. In the octagonal tiling, each hexagon contains one vertex taken from a "flipping pair», the flipping occuring under an infinitesimal shift of the band in $Z^{4}$ (in the cut and project method [4] language). Then, in the octagonal tiling, we only keep vertical and horizontal bonds, put bonds on the shorter diagonal of the thin rhombi, and link together two vertices in the same hexagon. The «labyrinth " is then obtained.

We found the last way to build the labyrinth in our attempt to find local growth rules for it, i.e. some rules allowing one two put a tile of the labyrinth, knowing the tiles in a circle of finite constant radius around it. Even if the labyrinth cannot be built by means of local growth 
rules, we can construct it with some local rules and only one non local rule. Let us start from four kites. Then, enumerating the possible local arrangements in the labyrinth, deduced from the properties of the Octonacci sequence, we found the local rules showed in figure 4 . We write $\mathrm{K}$ for a kite, $\mathrm{S}$ for a square and $\mathrm{T}$ for a trapezoid, and build the labyrinth for $r=1$, even if those rules are correct for any $r$ (with few modifications since the three tiles are then different). They have to be read in the following way : if we find one of the arrangements quoted in figure 4 (or this arrangement rotated by a multiple of $\pi / 2$ ), add letter(s) according to the rule corresponding to this arrangement. The procedure has to be carried out until it is no longer possible to put any other tile. Then, we apply the only non local rule needed : we add one tile on the closest free place to the $x$ and $y$ axes, and to the origin. There are eight such points. The tile is the same for those points, and is choosen in order that the sequence of large and short bonds between groups of four kites along the $x$ axis follows $\mathrm{S}_{\infty}$ (because of the self-similarity of the labyrinth). We show the first iterations of this method in figure 5. Of course, instead of applying this non local rule, we can decide to put a tile on a random free place, preserving the four-fold symmetry or not. Then, we obtain a labyrinth-like quasiperiodic tiling. In that case, some arrangements appear that cannot be found in the real labyrinth (Fig. 5). Moreover, in the random case, and when $r \neq 1$, there are some defects, since the $\mathrm{K}$ and the $\mathrm{T}$ are not squares any longer.

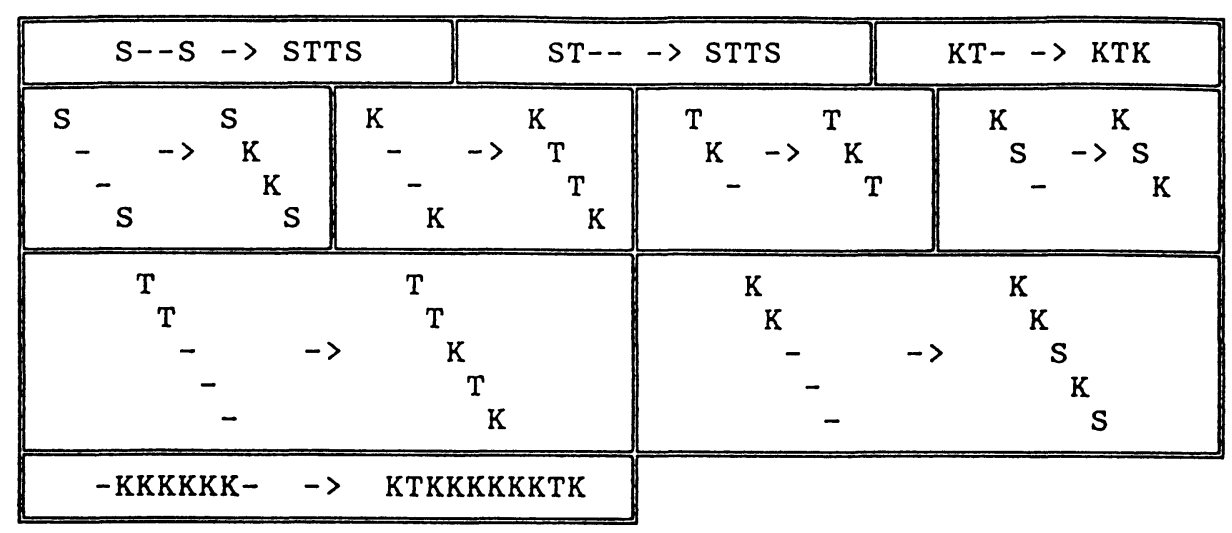

Fig. 4. - Local rules for the labyrinth on a square lattice $(r=1)$. « - represents a free place, « $K$ » kite, $\ll \mathrm{T} »$ a trapezoid, and $\ll \mathrm{S} »$ a square.

\section{Reciprocal space.}

In this section we are interested in the $k$-space of the labyrinth and especially to its symmetries. A well-known result concerning quasicrystals like the quasiperiodic octagonal tiling, is that their spectrum is dense. Moreover, in a bounded part of the reciprocal space, and for any $I_{0}>0$, one can find only a finite number of peaks whose intensity exceed $I_{0}$. Thus, experimentally, for real quasicrystal, one can only observe a point spectrum whose symmetries give us some informations about the structure into the real space.

We show below that,even if the labyrinth has only a true four-fold symmetry, it would have been very difficult to distinguish « experimentally » its spectrum from the spectrum of the octagonal tiling, which has a eight-fold symmetry. Let us now proceed to the calculation of the spectrum of the labyrinth. We showed in sections 2 and 3 that the points of the labyrinth are 


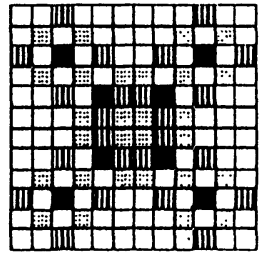

a)

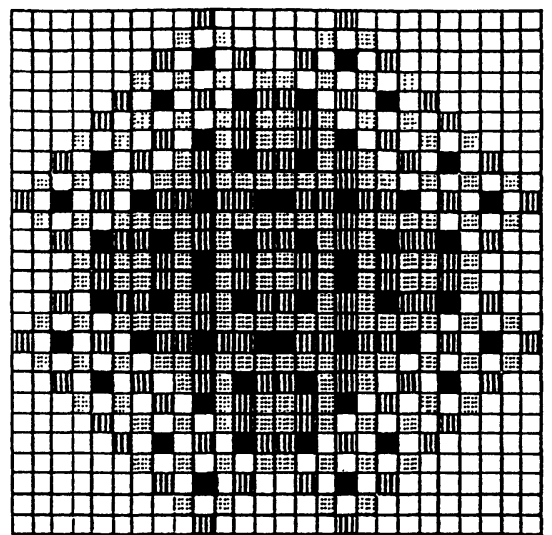

b)

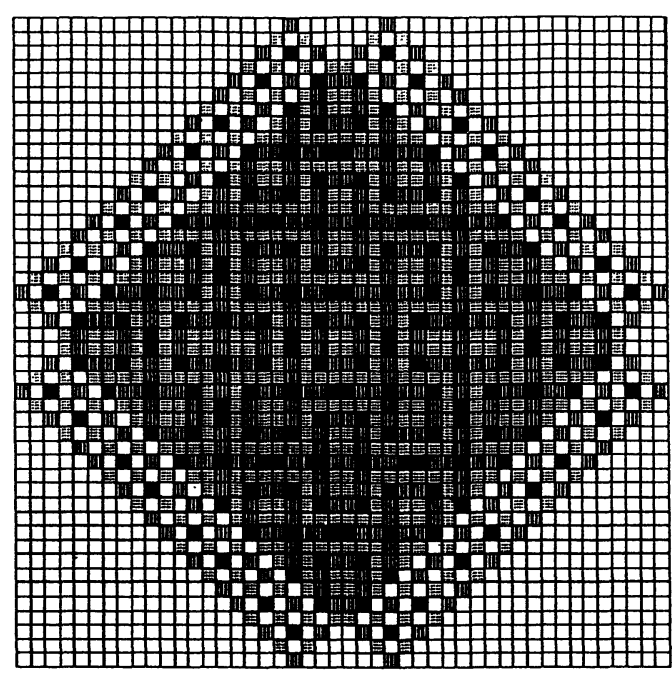

c)

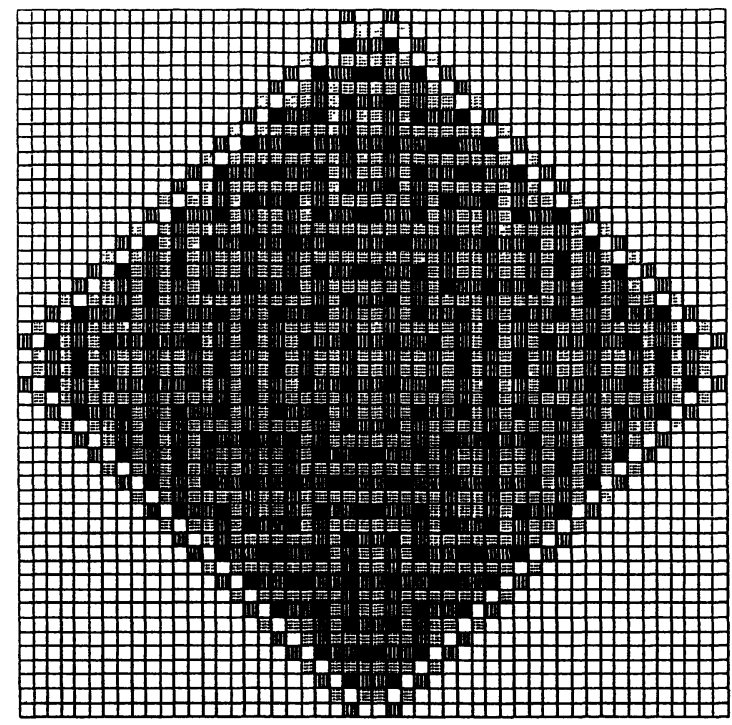

d)

Fig. 5. - (a)-(c) The first iterations of the procedure described in section 4. (d) An example, where the non local rule has been replaced by a random choice. (With the correspondence, square = black, kite $=$ grey and trapezoid $=$ stripes $)$.

located at the nodes of the product of two Octonacci linear chains whose coordinates have the same parity. Thus, we define $I(q)$ and $J(q)$, the Fourier transforms of both chains $\left\{U_{2 p}, p \in Z\right\}$ and $\left\{U_{2 p+1}, p \in Z\right\}$

$$
I(q)=1 \sum_{p=1}^{\infty} 2 \cos \left(2 \pi q U_{2 p}\right) \text { and } J(q) \sum_{p=0}^{\infty} 2 \cos \left(2 \pi q U_{2 p+1}\right) .
$$

and we have the following relation between $I, J$ and $S$, the Fourier tranisforms of the labyrinth

$$
S(\mathbf{k})=\left(I\left(k_{1}\right) I\left(k_{2}\right)+J\left(k_{1}\right) J\left(k_{2}\right)\right) / 2 \quad \mathbf{k}=\left(k_{1}, k_{2}\right)
$$


With this normalization, the intensity of the peak at $k=0$ is equal to 1 . We now derive the exact form of $I(q)$ and $J(q)$, by the mean of the cut and project method [4], which easily leads to the spectrum. From the form of $U_{p}$ we see that $S_{1}$ can be obtained by the projection of the points of $\mathrm{Z}^{2}$ belonging to a strip of slope $\Phi$ obtained by the translation of the unit square on a line of the same slope. Furthermore, since $U_{p}=-U_{-p}$, we deduce that the center of the unit square must be on vertex of the square lattice for the chain to be symmetric. In order to evaluate $I(q)$ (and $J(q)$ ), we sum the contributions of points, which are the projections of vertices in $\mathrm{Z}^{2}$ whose coordinates have the same parity (respectively, of the opposite parity). A straightforward calculation gives the intensities of the peaks of both even and odd chains :

$$
\begin{aligned}
& I\left(k_{m, n}\right)=\sin \left(H p_{m, n}\right) / p_{m, n} \\
& J\left(k_{m, n}\right)=(-1)^{m} \sin \left(H p_{m, n}\right) / p_{m, n} \quad \text { with } \quad m \equiv n[2]
\end{aligned}
$$

and

$$
k_{m, n}=m \cos (\theta) / 2+n \sin (\theta) / 2, \quad p_{m, n}=n \cos (\theta) / 2-m \sin (\theta) / 2
$$

where $H=\pi(\cos (\theta)+\sin (\theta))$, and $\theta$ is defined by $\tan (\theta)=\Phi$. Now we can deduce the intensity of the diffraction peaks of the labyrinth $W(\mathbf{k})=|S(\mathbf{k})|^{2}$ by using (5) and (6). It gives the diffraction pattern of figure 6. At first sight, it seems to have the eight-fold symmetry. We show in the following that it is not true, by studying the spectrum on the two axes $k_{2}=0$ and $k_{1}=k_{2}$, for which the calculations are quite easy. First, we show the following result : if $\mathbf{k}=(k, 0)$ is in the spectrum, then $\mathbf{k}^{\prime}=(k / \sqrt{2}, k / \sqrt{2})$ which is the vector $\mathbf{k}$ rotated by $\pi / 4$, is also in the spectrum. Indeed, if $\mathbf{k}=(k, 0)$ is in the spectrum of the labyrinth, then it is easy to show that the integers $m$ and $n$ in (6) must be even. Thus, $k$ can be written $k=m \cos (\theta)+n \sin (\theta)$ and we have to verify that $k / \sqrt{2}$ can be written in the same way as in (6). It is a consequence of the equality

$$
\begin{aligned}
k / \sqrt{2} & =\cos (\theta)(m \sqrt{2} / 2+n(1+\sqrt{2}) \sqrt{2} / 2) \\
& =\cos (\theta)((1+\sqrt{2})(n+m) / 2+(n-m) / 2) .
\end{aligned}
$$

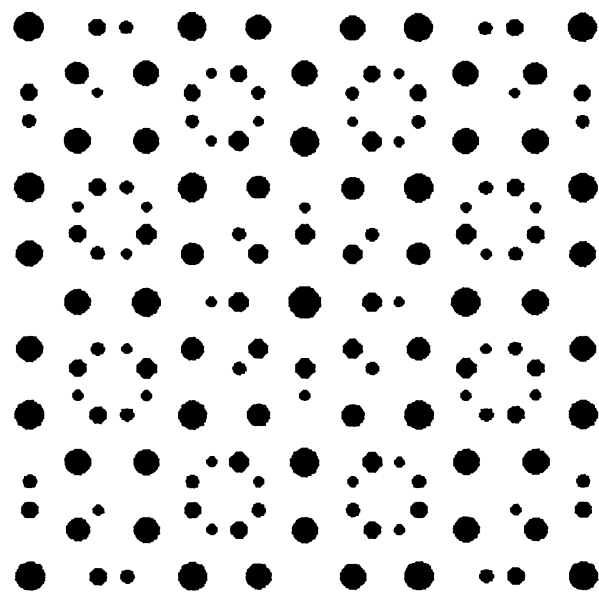

a)

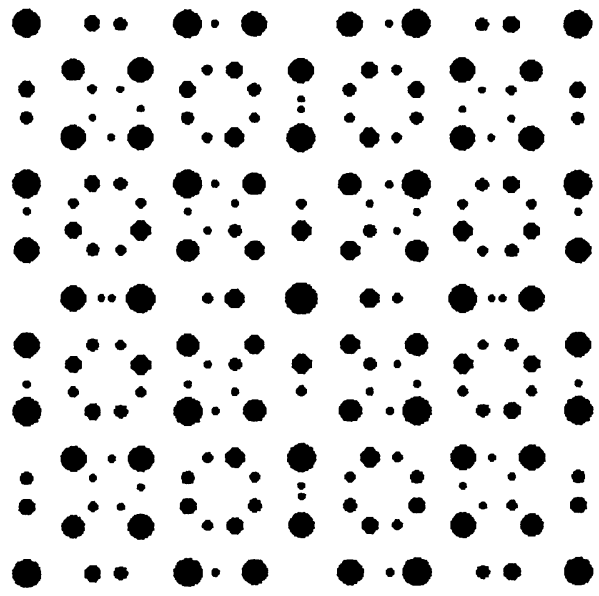

b)

Fig. 6. - Diffraction pattern of the labyrinth. The area of a spot is proportional to the square of $S(\mathbf{k})$. We have only considered peaks whose intensity were greater than $I_{0}$, with the convention that the intensity at $\mathbf{k}=0$ is equal to 1 . For $I_{0}$, we have taken, (a) $I_{0}=0.06$, (b) $I_{0}=0.04$. 
Moreover, this property is not only true for $\mathbf{k}$ on the $x$ axis, but for every $\mathbf{k}$ in the spectrum, what can be seen by a calculation of the same kind as above. The strict four-fold symmetry of the Fourier spectrum was expected since in the cut and project method, the labyrinth acceptance region is a square tangent to the octagonal region corresponding to the octagonal tiling [11]. We have to notice that it can happen that the peak at $\mathbf{k}^{\prime}$ vanishes, it the phase in the sine in (6) is a multiple of $2 \pi$. We find that it occurs when $k$ is of the form $k=2 n(\cos (\theta)+\sin (\theta))=n \sqrt{(4+2 \sqrt{2})}$ where $n$ is an integer. But for those values of $\mathbf{k}$, we shall see that the intensity in $\mathbf{k}$ is only $4.7 / n^{2}$ percent of the intensity at $\mathbf{k}=0$. This property of invariance by a rotation of $\pi / 4$ (even if the intensities are not the same) is shared by the quasiperiodic tilings built from an Octonacci like sequence but no other sequence. For example, the $2 \mathrm{D}$ quasicrystal built with the Fibonacci sequence does not show the same property. Being the product of two linear chains, its spectrum has of course the fourfold symmetry. But, if $\mathbf{k}$ is in the spectrum, then the vector $\mathbf{k}$ rotated by $\pi / 4$ never belongs to it. Now, going back to the labyrinth, we can compare the two intensities at $\mathbf{k}$ and $\mathbf{k}^{\prime}$. Let us define

$$
\begin{aligned}
R(k) & =W(k)-W\left(k^{\prime}\right) \\
& =I\left(p_{k}\right)^{2}-I\left(p_{k} / \sqrt{2}\right)^{4} \\
& =\sin \left(H p_{k}\right)^{2} /\left(H p_{k}\right)^{2}-4 \sin \left(H p_{k} / \sqrt{2}\right)^{4} /\left(H p_{k}\right)^{4}
\end{aligned}
$$

where we define $p_{k}=n \cos (\theta)-m \sin (\theta)$ for $k=m \cos (\theta)+n \cos (\theta)$. If we look for $R_{\max }$ the absolute maximum of $|R(k)|$ we find

$$
R_{\max } \approx 0.047 \quad \text { for } \quad p_{\max } \approx 1.095 \text {. }
$$

So, the maximum disagreement between both spectra arises for the value

$$
\begin{aligned}
& p=2(\cos (\theta)-\sin (\theta))=\sqrt{(4-2 \sqrt{2})} \approx 1.083 \\
& k=2(\cos (\theta)+\sin (\theta))=\sqrt{(4+2 \sqrt{2})}
\end{aligned}
$$

where $W(\mathbf{k}) \approx 0.047$ and $W\left(\mathbf{k}^{\prime}\right)=0$. This value is easy to understand : since the labyrinth is obtained by adding vertices to the octagonal quasiperiodic tiling we expect its spectrum to ressemble that of the octagonal tiling. More precisely we expect the maximum disagreement to be about the square of the number of points we add per « atom » of the octagonal tilling, that is $(1 / 2-1 / \sqrt{2})^{2} \approx 0.043$, which is quite close to the value effectively obtained. Moreover if the intensity $W(\mathbf{k})$ is quite large then $W\left(\mathbf{k}^{\prime}\right)$ will be very close to $W(\mathbf{k})$. This explains the «quasi » eight-fold symmetry of the labyrinth. For example, for $k=\cos (\theta)+3 \sin (\theta)$, we have $W(\mathbf{k}) \approx 0.748$ and $W\left(\mathbf{k}^{\prime}\right) \approx 0.751$, and for $\mathrm{k}=\cos (\theta)+2 \sin (\theta)$, we find $W(\mathbf{k}) \approx 0.8667$ and $W\left(\mathbf{k}^{\prime}\right)=0.8674$. If we do the same study with $\mathbf{k}$ not on the $x$ axis, we obtain very similar results (compare Figs. 6a and b).

Thus, in this section we have shown an explicit example of a quasiperiodic tiling, whose spectrum seems at first sight to have the eight-fold symmetry, without having it perfectly. Furthermore, the «experimental » spectrum of the labyrinth would have been said to have the eight-fold symmetry, if the resolution were at best $5 \%$. This raises the question of whether or not it is really possible to specify at the experimental level, the real symmetries of a quasicrystal. The problems encountered at the moment, to propose icosahedral quasiperiodic structures which fit exactly the experimental diffraction patterns, are perhaps linked to the problem developed in this section. Can we find modelized quasicrystals which have not the icosahedral symmetry, but whose spectrum fits the experimental patterns at the accuracy of the measures? Note that this question differs from the controversy regarding the 
description of the experimental aperiodic phases, either as quasicrystals, or crystals with large unit cell. Indeed, while in that case the diffraction spectrum sit at different points (even very close), in our case, the locations of points are the same, only do the intensities slightly vary.

\section{Recovering the octogonal quasicrystal.}

Now, we show the way to recover the octagonal quasiperiodic tiling from the labyrinth whereas, in section 3, we have shown how to build the labyrinth from the standard octagonal quasiperiodic tiling. If we look for the possible decorations of each tile of the labyrinth (Fig. 7), we only find one decoration for the kite and the square, and two possible decorations for the trapezoid, which are always coupled (Fig. 2). Moreover, since the square can have four orientations, we have to show that the degrees of freedom due to the two possible decorations of the trapezoid, and the four possible orientations of the square are only apparent. In order to recover the octagonal tiling, we first decorate every kite of the labyrinth in the way shown in figure 2 . We have already noted that every path of kites is closed. At each

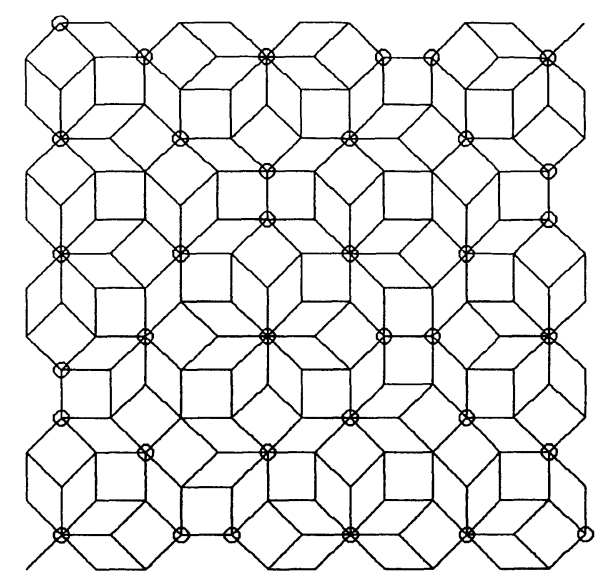

a)

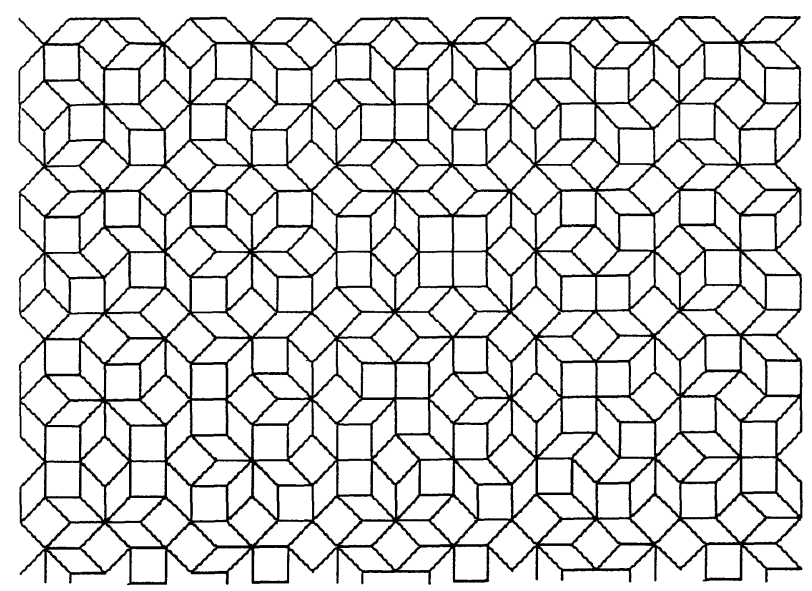

b)

Fig. 7. - (a) The labyrinth as obtained by the method of section 3. (b) An octogonal-like quasiperiodic tiling generated by means of the random procedure described in section 6 . 
corner of those paths, there is only one way to decorate a square. Following the path of kites there is only one way to decorate the trapezoid after the square. The next trapezoid is choosen symmetric to the last one. Then, there is only one way to decorate the next square with respect to the decorations of the last trapezoid and the border of kite except if the square is surrounded by four trapezoids. In that case we have to wait until we know the decoration of a trapezoid perpendicular to the direction of the trapezoid preceding the square, that we already know. We carry on the process until we find another corner, and so on. Thus, we find only one way to decorate the squares and trapezoids between two closed paths of kites in order to obtain the octagonal tiling. However, if we do not want to recover the exact octagonal tiling, but only a quasicrystal built with the same two tiles (the square and the rhombus), we can apply the following rules. Starting from the labytinth, remove one of the vertices of the shortest side of each trapezoid, and link the remaining points with bonds of the same length as the square side in the labyrinth. One can choose the point to remove, randomly or with any other rules. For example, among the two points, for a given trapezoid, we can remove the left-hand vertex, for horizontal bonds, and the bottom vertex, for vertical bonds. In the random case we expect the diffraction pattern to have a eight-fold symmetry, whereas in the second case the spectrum has only a four fold symmetry. Moreover, in general, we obtain tilings with certain local configurations that cannot be found in the real octagonal tiling (Fig. 7).

\section{Conclusion.}

In this paper, we have studied the geometric properties of a new quasiperiodic tiling, the « labyrinth », closely related to the well-known octagonal tiling. We showed a recursive way to build the labyrinth, and then, gave explicit coordinates for its vertices. The labyrinth has an average lattice, which is a square lattice. It is self-similar but cannot be built by means of local growth rules. Moreover, its diffraction pattern exhibits an interesting property. Although it has strictly speaking, only the four-fold symmetry, its spectrum has « nearly » the eight-fold symmetry. Consequently, it would have been very difficult to make the difference « experimentally» (if we have not known its spectrum analytically).

In a forthcoming paper, the excitation spectrum of the labyrinth, which possesses very interesting properties, will be presented [12].

\section{Acknowledgements.}

We are indebted to $\mathrm{M}$. Duneau and C. Oguey for stimulating conversations.

\section{References}

[1] Shechtman I., Blech I., Gratias D., Cahn J. W., Phys. Rev. Lett. 53 (1984) 1951.

[2] Sadoc J. F. and Mosseri R., to be published in J. Phys. France.

[3] Duneau M. and Oguey C., Number Theory and Physics, Les Houches, March 1989 (Springer), to be published.

[4] Duneau M. and Katz A., Phys. Rev. Lett. 54 (1985);

Kalugin P. A., Yu Kitaev A. and Levitov L. S., Pis'ma Zh. Eksp. Teor. Fiz. 41 (1985) 119 ;

Elser V., Phys. Rev. Lett. 54 (1985) 1730.

[5] BeEnKer F. P. M., report 82-WSK-04, Sept. 82, Dept. of Math. (Un. of Technology, Eindhoven).

[6] Socolar J. E. S., Phys. Rev. B 39 (1989) 10519, for recent aspects.

[7] Aubry S., Godrèche C., Vallet F., J. Phys. France 48 (1987) 327. 
[8] Oguey C., Duneau M., to be published.

[9] Friedel J., Denoyer F., C. R. Acad. Sc. Paris 305 (27) 171.

[10] Makler S. S., Gaspard J. P., to be published.

[11] DunEaU M., private communication.

[12] SiRE C., to be published in Europhys. Lett. (1989). 